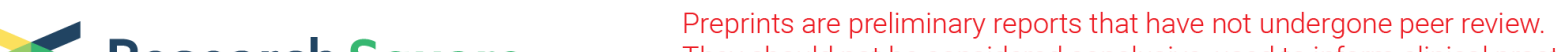 Research Square They should not be considered conclusive, used to inform clinical practice, or referenced by the media as validated information.
}

\section{An Improved Redundant Robotic Arm Parameter Identification Method}

\section{Shi-Ping Liu}

Huazhong University of Science and Technology School of Mechanical Science and Engineering

Ziyan Ma (D954247275@qq.com)

Huazhong University of Science and Technology https://orcid.org/0000-0003-0730-7104

\section{Jin-Liang Chen}

Huazhong University of Science and Technology School of Mechanical Science and Engineering Jun-Feng Cao

Huazhong University of Science and Technology School of Mechanical Science and Engineering Yan Fu

Huazhong University of Science and Technology School of Mechanical Science and Engineering Shi-Qi Li

Huazhong University of Science and Technology School of Mechanical Science and Engineering

\section{Original Article}

Keywords: redundant robotic arm, dynamic parameter identification, non-linear optimization

Posted Date: March 31st, 2020

DOI: https://doi.org/10.21203/rs.3.rs-19727/v1

License: (c) (i) This work is licensed under a Creative Commons Attribution 4.0 International License. Read Full License 


\section{An Improved Redundant Robotic Arm Parameter Identification Method}

Shi-Ping Liu, is currently an associate professor in the School of Mechanical Science and Engineering, Huazhong University of Science and Technology, China. His research interests include machine vision, intelligent robotics and Construction robotics.

Tel: +86-027-87541034; E-mail: liushiping@ hust.edu.cn

Zi-Yan Ma, is currently a master student in the School of Mechanical Science and Engineering, Huazhong University of Science and Technology, China.

E-mail:imaleilife@126.com

Jin-Liang Chen, is currently an engineer and he received a master's degree in mechanical engineering from Huazhong University of Science and Technology in China in 2019.

Jun-Feng Cao, is currently a master student in the School of Mechanical Science and Engineering, Huazhong University of Science and Technology, China.

Yan Fu, is currently an associate professor in the School of Mechanical Science and Engineering, Huazhong University of Science and Technology, China.

Shi-Qi Li, is currently an professor in the School of Mechanical Science and Engineering, Huazhong University of Science and Technology, China.

\section{Corresponding author: Zi-Yan Ma E-mail: imaleilife@126.com}




\title{
An Improved Redundant Robotic Arm Parameter Identification Method
}

\author{
Shi-Ping Liu • Zi-Yan Ma•Jin-Liang Chen • Jun-Feng Cao • Yan Fu • Shi-Qi Li
}

\begin{abstract}
In order to establish the dynamic model of redundant robotic arm more accurately, this paper studies the identification method of the dynamic parameters of redundant robotic arm. A method of introducing the theory of spins based on the Newton-Euler dynamics equations, and using the Coulomb + viscous friction model to further derive the minimum parameter set through linearization is proposed. The method of offline identification of current measurement is used to realize the parameter identification of the robotic arm. The excitation trajectory is designed in the basic form of Fourier series. The non-linear optimization function is used to optimize the coefficient of the trajectory with the minimum number of conditions in the observation matrix as the goal Therefore, the available joint trajectories are obtained, and the obtained parameter set has high accuracy and can meet the needs of actual control. Finally, the method was experimentally tested by actual robot Walker, which verified the accuracy and effectiveness of the proposed method.
\end{abstract}

Keywords: redundant robotic arm • dynamic parameter identification $\bullet$ non-linear optimization

\section{Introduction}

The identification of the dynamic parameters of the robotic arm mainly studies the parameter estimation methods of the structural parameters, inertial parameters and friction parameters of the robotic arm, and these parameters are the basis of the research of the robotic arm. In general, some structural parameters can be obtained through kinematic calibration, but dynamic parameters need to estimate by identification method[1-3]. At the same time, due to changes in the external working environment, the length of use of the working robotic arm, and the difference in the clamping equipment at the end of the working robotic arm will affect the dynamic characteristics of the robotic arm

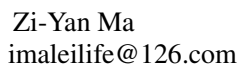

School of Mechanical Science \& Engineering,Huazhong University of Science and Technology, Wuhan 430074, China.
[4-5]. In order to obtain the torque calculation accuracy that meets the requirements and to achieve high-precision control based on the dynamic model without increasing the complexity of the dynamic model of the robotic arm, the research on parameter identification of the mechanical arm is very important.

At present, the research on dynamic parameter identification is divided into offline identification and online identification from the aspect of determining dynamic parameters, and it is divided into current measurement identification and torque measurement identification in terms of torque measurement[6-8]. Online identification is to find the parameter values in the mathematical model of the system from the online measured data, so that the predicted dynamic response is consistent with the response of the actual system, Jubien [9], Gaz [10-11], Burdet and Codourey [12], Honegger et al [13], all realize the parameter identification of robotic arm through online identification methods. While scholars such as Jiang [14] found that it can also be effectively used for online identification of nonlinear systems by studying neural networks, Ghannadi et al [15] applied DPI to parameter identification of robots with actuator gaps. The offline identification is different from the parameter value calculation based on the online measured data. The parameter identification of the robotic arm can be realized mainly through physical experimental methods, computer-aided design (CAD) methods, and trajectory motion methods [16]. TourassisVD et al [17] used physical experiments to determine the inertial parameters by using the vibration response of the connecting rod. Brown and others [18] used friction models to identify the parameters, Guegan et al [19] and Vivas et al [20] all realized the parameter identification of the robotic arm by planning the trajectory of the robotic arm. The basis of current measurement identification is the linear relationship between the driving torque of the motor and the current. Gautier [21] uses Kalman filtering and least squares 
method to identify the parameters of the robotic arm when the current data is used to identify the parameters. The results are satisfactory. The key to the realization of force measurement identification is the collection of force sensing information of the robot. Guaniun Liu , Karl Iagnemma [22] proposed a method of identification of inertial parameters based on force measurement, and the dynamic parameters of the robotic arm were obtained through calculation.

Robot parameter identification is one of the key issues for accurate robot modeling and robot control and simulation. It has attracted much attention from scholars at home and abroad. With the expansion of the application scope of robots, higher requirements are placed on the control accuracy of robots. More accurate parameter identification methods are an important basis for improving control accuracy. The current parameter identification methods still have disadvantages such as inability to simulate complex influencing factors and low identification accuracy[23-25]. In the research of this paper, the spin theory is introduced on the basis of the Newton-Euler dynamics equation. The minimum parameter set is obtained by linearization derivation taking into account the friction term. Design the excitation trajectory in the basic form of a Fourier series, taking full account of joint limits and joint motion speed, The non-linear optimization function [26] is used to optimize the coefficients of the excitation trajectory by minimizing the condition number of the observation matrix to obtain the available joint trajectories. Finally, the correctness and effectiveness of the method are verified on an actual robot.

\section{Parameter Identification Principle of Redundant Robotic Arm}

\subsection{Dynamic Model of Redundant Robotic Arm Considering Friction}

The dynamic model in this paper is based on the Newton-Euler equation. Since the redundant mechanical arm studied only has a rotating joint, it can be simplified to express it without friction and external force terms:

$$
\tau=M(q) \ddot{q}+C(q, \dot{q}) \dot{q}+G(q)
$$

In the formula, $M(q)$ represents inertial force term, $C(q, \dot{q})$ represents centripetal force and Coriolis force term, and $G(q)$ represents gravity term.

In the actual control of a robotic arm, friction is a key factor that affects the accuracy of the control. Therefore, in the parameter identification, the identification of the friction parameter is very important. It is further generalized by equation (1). The dynamic equation considering the existence of the friction term is:

$$
\tau=M(q) \ddot{q}+C(q, \dot{q}) \dot{q}+G(q)+\tau_{f}
$$

In the formula, $\tau_{f}$ is the friction term. In actual identification, the classical Coulomb + viscous friction model is adopted for the establishment of the friction model, and the specific form is as follows:

$$
\tau_{f}= \begin{cases}f_{c} \operatorname{sign}(\dot{q})+f_{v} \dot{q} & |\dot{q}| \geq \dot{q}_{\min } \\ 0 & |\dot{q}|<\dot{q}_{\text {min }}\end{cases}
$$

In the formula, $f_{c}$ is the Coulomb friction constant, $f_{v}$ is the viscous friction constant, and $\dot{q}_{\min }$ is the speed threshold of static friction to dynamic friction in joint motion.

\subsection{Set of Minimum Inertia Parameters}

In the identification of the dynamic parameters of the robotic arm, the structural parameters and inertial parameters of the joints need to be considered while considering the friction model. The simple configuration of the joint link is shown in Figure 1 below.

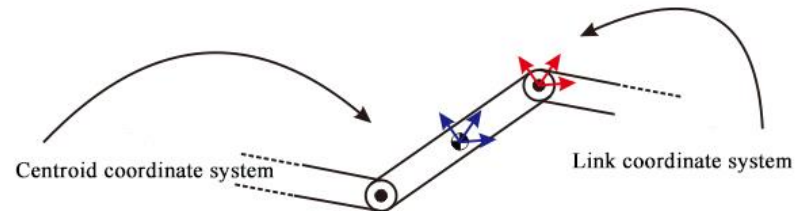

Figure 1 simplified diagram of joint parameters

It is clear from this that for each joint link $k$, its parameters include mass $m_{k}$, the center of mass position $r_{k}$ in the link coordinate system, the first moment of inertia $l_{k}$, and the inertia tensor $L_{k}$ in the link coordinate system, from this:

$$
l_{k}=m_{k} r_{k}
$$

At the same time, according to Huygens-Steiner theorem, we can get:

$$
L_{k}=I_{k}+m_{k} S^{T}\left(r_{k}\right) S\left(r_{k}\right)
$$

In the formula, $I_{k}$ is the centroid inertia tensor, and $S\left(r_{k}\right)$ is the skew-symmetric matrix operator of $r_{k}$.

Existing studies have shown that equation (2) can be linearized as follows through the combination and 
transformation of parameters:

$$
H(q, \dot{q}, \ddot{q}) \delta=\tau
$$

For equation (6), dynamic parameters can be identified through linear regression, so it can be written as follows:

$$
H_{S} \delta \simeq \omega
$$

Among them, $\mathrm{H}_{\mathrm{s}}$ is a regression matrix composed of the superposition of the joint pose (joint angle, joint speed, and joint angular acceleration) of the robotic arm, expressed as follows:

$$
H_{S}=\left[\begin{array}{c}
H\left(q_{1}, \dot{q}_{1}, \ddot{q}_{1}\right) \\
H\left(q_{2}, \dot{q}_{2}, \ddot{q}_{2}\right) \\
\vdots \\
H\left(q_{S}, \dot{q}_{S}, \ddot{q}_{S}\right)
\end{array}\right]
$$

And $\omega$ is a matrix composed of the measured moments corresponding to these joint poses, expressed as follows:

$$
\omega=\left[\begin{array}{c}
\tau_{1} \\
\tau_{2} \\
\vdots \\
\tau_{S}
\end{array}\right]
$$

Generally speaking, if there is a torque sensor at the joint of the robot arm, it can be read directly. When there is no torque sensor, the joint motor current data is used. Among them, $\delta$ is the dynamic parameter set of the link of the robot arm. For a robot arm system with the number of links $\mathrm{N}$, its parameter set is as follows:

$$
\delta=\left[\begin{array}{llllll}
\delta_{1}^{T} & \delta_{2}^{T} & \cdots & \delta_{k}^{T} & \cdots & \delta_{N}^{T}
\end{array}\right]^{T}
$$

For each $\mathrm{k}$ link of the mechanical arm, its parameter set $\delta$ consists of mass, inertia tensor, first moment of inertia, and friction parameters, as follows:

$\delta_{k}=\left[\begin{array}{cccccccc}L_{k, x x} & L_{k, x y} & L_{k, x z} & L_{k, y y} & L_{k, y z} & L_{k, z z} & \cdots \\ & l_{k, x} & l_{k, y} & l_{k, z} & m_{k} & f_{v k} & f_{c k} & \end{array}\right]^{T}$

The link parameter set $\delta$ can be estimated by linear regression based on the minimum residual $\varepsilon$, as follows:

$$
H_{S} \delta+\varepsilon=\omega
$$

In actual calculations, because some parameters have no effect on the robot dynamics, and other parameters have a linear proportional relationship, the matrix $H_{S}$ has zero columns and linear correlation columns, which results in $H_{S}$ being a singular matrix with multiple solutions. To solve this problem, the parameters are usually eliminated and recombined into the basic parameter vector $\beta$, expressed as follows:

$$
\beta=\delta_{b}+K_{d} \delta_{d}
$$

In the formula, $\delta_{b}=P_{b}^{T} \delta, \delta_{d}=P_{d}^{T} \delta$, where $P_{b}^{T}, P_{d}^{T}$ truncates the permutation matrix, $\delta_{b}$ and $\delta_{d}$ are independent parameters and related parameters selected from $\delta ; K_{d}$ is a dependency matrix in which related parameters are grouped into independent parameters. As follows:

$$
W \beta+\varepsilon=\omega
$$

In the formula, $W$ can be obtained by eliminating the correlation column of $H_{S}$.At this time, the regression problem can be expressed as follows:

$$
\underset{\beta}{\operatorname{minimize}}\|\omega-W \beta\|^{2}
$$

The optimal solution of the above equation is expressed as follows:

$$
\hat{\beta}=\left(W^{T} W\right)^{-1} W^{T} \omega
$$

At this time, $\hat{\beta}$ is the required minimum parameter set.

\section{Experimental verification}

\subsection{Design and Optimization of Excitation Trajectory}

When identifying the parameters of the robotic arm, the design of the excitation trajectory is very important. The excitation trajectory should not only fully stimulate the dynamic parameters of the robotic arm, but also reduce the influence of noise in the measurement as much as possible. it can not only estimate the parameters quickly and accurately, but also ensure the accuracy of identification. For the excitation trajectory, the velocity constraint and acceleration constraint in the joint motion should also be fully considered in the design to ensure that the sudden change of the joint velocity and acceleration in the joint 
motion is as small as possible. Many scholars have carried out extensive research. The excitation trajectory used in this paper is based on the basic form of periodic Fourier series. The excitation trajectory based on periodic Fourier series in the parameter identification of robotic arm is as follows:

$$
q_{k}(t)=q_{k 0}+\sum_{l=1}^{L} \frac{a_{k, l}}{\omega_{f} l} \sin \left(\omega_{f} l t\right)-\sum_{l=1}^{L} \frac{b_{k, l}}{\omega_{f} l} \cos \left(\omega_{f} l t\right)
$$

In the formula, $a_{k, l}, b_{k, l}$ is the Fourier series coefficient, $\omega_{f}$ is the fundamental frequency of Fourier series, $L$ is the number of harmonics, and $q_{k 0}$ is the initial position of the excitation trajectory in joint identification.

Because of the coefficient of Fourier series, the value of $a_{k, l}, b_{k, l}$ has a great influence on the overall performance of the excitation trajectory, so it is necessary to optimize the $a_{k, l}, b_{k, l}$ parameters. In this paper, the condition number of the observation matrix is taken as the optimization objective, and the condition number of the matrix is the quantitative expression of whether the matrix is morbid or not. the larger the condition number of the matrix is, the more sensitive the matrix calculation is to the small disturbance. in dynamic parameter identification, that is, the larger the condition number of $W$ matrix, the small error of joint torque measurement $\omega$ will also lead to the inaccuracy of dynamic parameter identification. the specific optimization problems are described as follows:

$$
\begin{aligned}
& \min _{q_{k 0}, a_{k, l}, b_{k, l}} \operatorname{cond}(W) \\
& \text { subject to } \\
& q(0)=q_{\text {init }}, \dot{q}(0)=0, \ddot{q}(0)=0 \\
& q\left(t_{f}\right)=q_{\text {final }}, \dot{q}\left(t_{f}\right)=0, \ddot{q}\left(t_{f}\right)=0 \\
& q_{\min } \leq q(t) \leq q_{\max }, \dot{q}_{\min } \leq \dot{q}(t) \leq \dot{q}_{\max } \\
& \ddot{q}_{\min } \leq \ddot{q}(t) \leq \ddot{q}_{\max }
\end{aligned}
$$

In the formula, $q_{\text {init }}, q_{\text {final }}$ is the initial angle and end angle of the joint; $q_{\min }, q_{\max }$ is the upper and lower limit of the joint angle in the motion of the robotic arm; $\dot{q}_{\text {min }}, \dot{q}_{\text {max }}$ is the upper and lower limit of the angular velocity of the joint in the motion of the robotic arm; $\ddot{q}_{\text {min }}, \ddot{q}_{\text {max }}$ is the upper and lower limit of the angular acceleration of the joint in the motion of the robotic arm.
The coefficient optimization of Fourier series is solved by the nonlinear constrained optimization function fmincon in Matlab, and the iterative optimization is carried out by setting the initial angle of the joint and the range of angle, angular velocity and angular acceleration in the joint motion. For the seven-degree-of-freedom robotic arm, the condition number of the $\mathrm{W}$ matrix is generally optimized to less than 100 and converges at the same time. The number of iterations in this optimization is 164 , and the condition number of the final optimized $\mathrm{W}$ matrix is 75.5086 . The change of the condition number in the optimization process is shown in figure 2. The values of fourier series coefficients obtained by nonlinear function optimization $a_{k, l}, b_{k, l}$ are shown in Table 1.

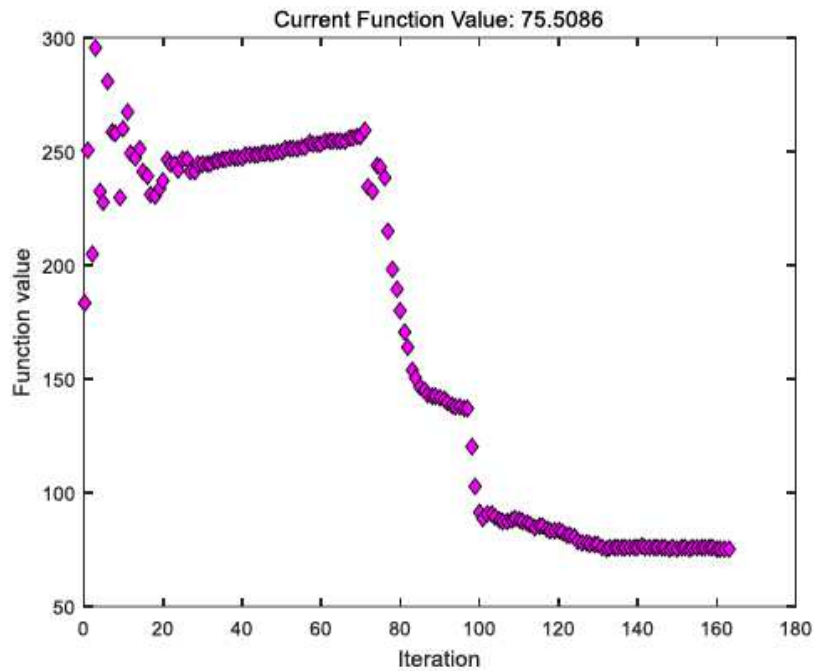

Figure 2 the change of condition number in the process of optimizing Fourier series coefficients

Table 1 Fourier series coefficients obtained by optimization

\begin{tabular}{cccccccc} 
parameter & joint1 & joint2 & joint3 & joint4 & joint5 & joint6 & joint7 \\
\hline \multirow{6}{*}{$a_{k, l}$} & 1.1318 & -1.4863 & -0.35916 & -2.7582 & -0.99332 & -0.34652 & -2.369 \\
& 0.42525 & -0.71812 & 0.12917 & 0.52948 & -1.2149 & 0.24585 & 1.3629 \\
& 0.81248 & 1.0781 & -0.29338 & -0.71537 & 1.4274 & -1.5854 & 0.27264 \\
& -1.2358 & -0.64219 & 2.0571 & -0.58536 & 0.021041 & -1.8848 & -1.0537 \\
& -1.1337 & 1.7684 & -1.5337 & 3.5295 & 0.75979 & 3.5709 & 1.7871 \\
\hline \multirow{6}{*}{$b_{k, l}$} & 0.090313 & -0.71072 & 0.098464 & -0.10925 & -0.11717 & 0.69369 & -0.71614 \\
& -1.1092 & 0.080641 & -1.5471 & 0.29085 & 1.1663 & -1.6948 & 1.0262 \\
& -0.58771 & 0.8885 & -0.27425 & -0.14694 & -0.4319 & -0.18596 & 1.4161 \\
& 0.90503 & -0.15831 & 1.3792 & 0.89334 & 0.96067 & 0.004065 & -1.3927 \\
& 2.1697 & 2.0691 & 2.1086 & -1.0527 & -2.8109 & 1.0734 & 0.39577 \\
\hline
\end{tabular}

\subsection{Trajectory Tracking and Motion Data Sampling}

According to the Fourier series coefficients obtained, the value of $a_{k, l}, b_{k, l}$ can further obtain the joint trajectories of the seven joints of the redundant robotic arm, that is, the angle changes (radians) of each joint in a single motion 
period $(20000 \mathrm{~ms}$, that is, $20 \mathrm{~s})$. In actual identification, the excitation trajectory of more than three cycles is generally run to ensure that sufficient joint data are collected for calculation and processing.

In the process of joint motion, the data are sampled at the time interval of $10 \mathrm{~ms}$, and the data contents are time point, joint angle, joint angular velocity, joint angular acceleration and joint torque. the general robotic arm joint can read the joint torque data directly by the torque sensor, if there is no sensor rigid joint can read the motor current value, and the parameter identification of the robotic arm can be realized by measuring the current. However, the joint angular velocity data can not be read directly, and it is generally calculated by the differential of joint angular velocity.

\subsection{Parameter Estimation and Inverse Dynamics \\ Verification}

The off-line identification of the dynamic parameters of the robotic arm is carried out according to the collected data. Firstly, the data is preliminarily processed by MATLAB, the data points are improved by one-dimensional data interpolation to reduce the time node, and then the moving average filter is used to smooth the data to deal with the noise data. According to the data acquisition, multiple filtering can be carried out on the basis of one filter. Because the design of the excitation trajectory is composed of the basic form of Fourier series, it is not sensitive to noise data, and the needed data can be easily obtained through processing, so only secondary filtering is carried out in this study. the joint torque data is shown in figure 3 , in which the green line is the original data, the blue line is the primary filtering data, and the red line is the secondary filtering data. In the actual data processing, because the data collected in the early stage will vibrate because the robotic arm just started to move at a low speed, so the data of the first 20 seconds will be left out for research.

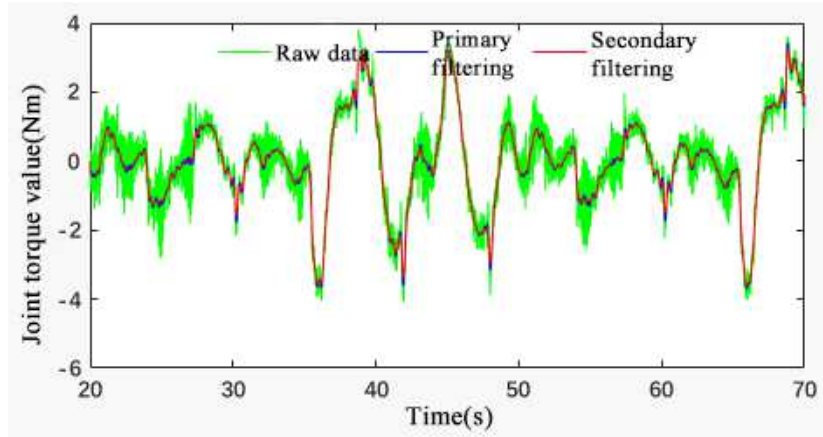

(a) Joint 1

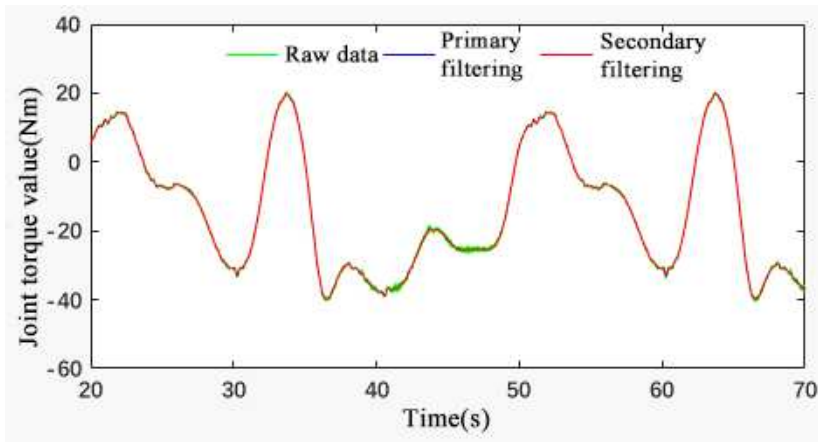

(b) Joint2

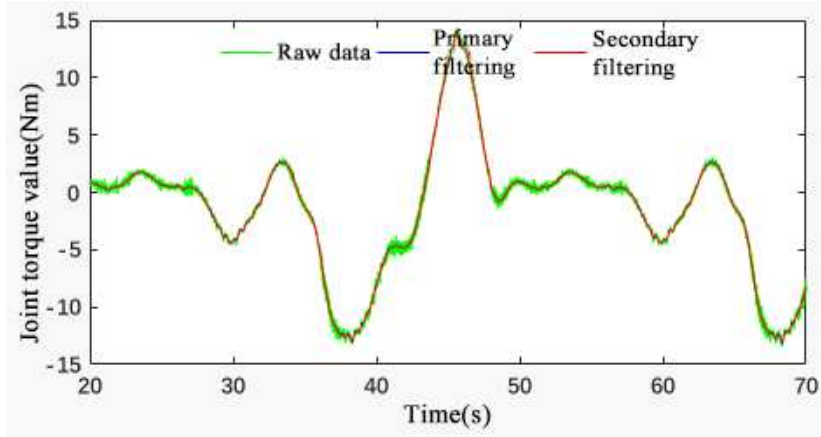

(c) Joint3

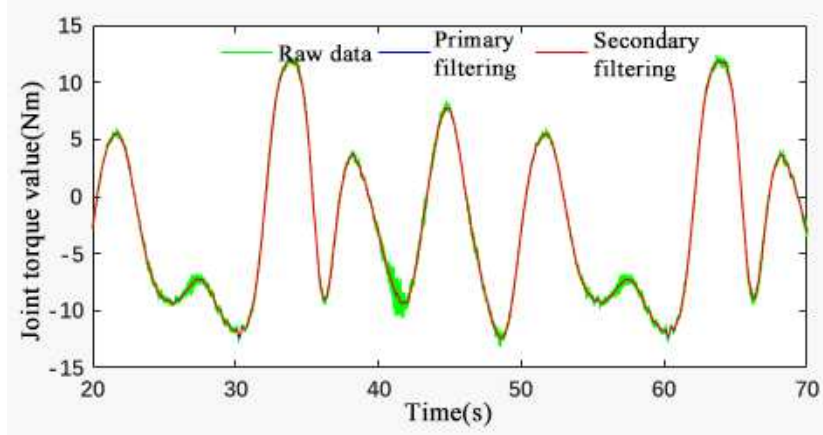

(d) Joint4

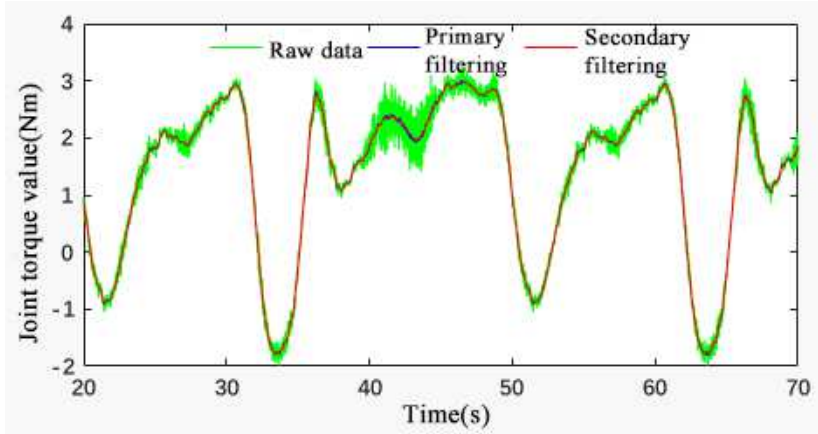

(e) Joint5 


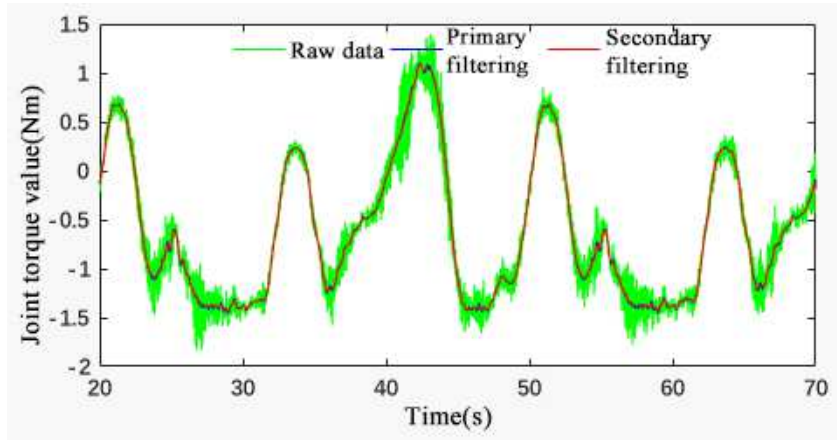

(f) Joint6

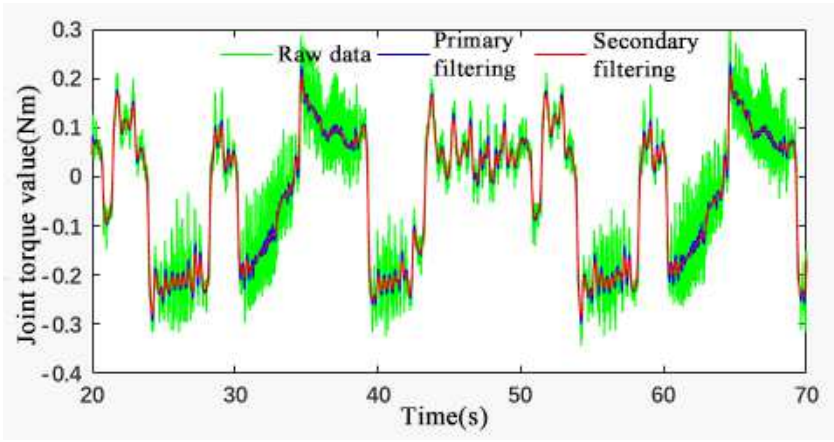

(g) Joint7

Figure 3 joint torque value after data processing

In the process of identification, the equation (7) can be obtained by linearizing the dynamic model according to the previous theoretical basis, and the specific parameters are identified and deduced, and the final identification result is obtained:

$$
\hat{\beta}_{\text {OLS }}^{\prime}=\left(R_{1}^{T} R_{1}\right)^{-1} R_{1}^{T} \rho_{1}
$$

The above is a further derivation of the calculation of the minimum parameter set according to the requirements of the actual programming calculation. According to the above formula, the minimum parameter set of redundant robotic arm under Coulomb + viscous friction model is calculated by Matlab programming. At this time, the inverse dynamics verification is carried out with the same original data, and the calculated torque value $\omega$ can be obtained by substituting the minimum parameter set into the equation (7). Further, by analyzing the accuracy of the identification result by analyzing the difference between the calculated torque value and the actual torque value, the joint torque data is shown in figure 4 , in which the green line is the actual value of the joint torque, that is, the actual torque value, and the blue line is the calculated value of the joint torque. That is, the calculated torque value is calculated according to the linearization equation of the dynamic model and the minimum parameter set, and the red line is the calculation error between them.

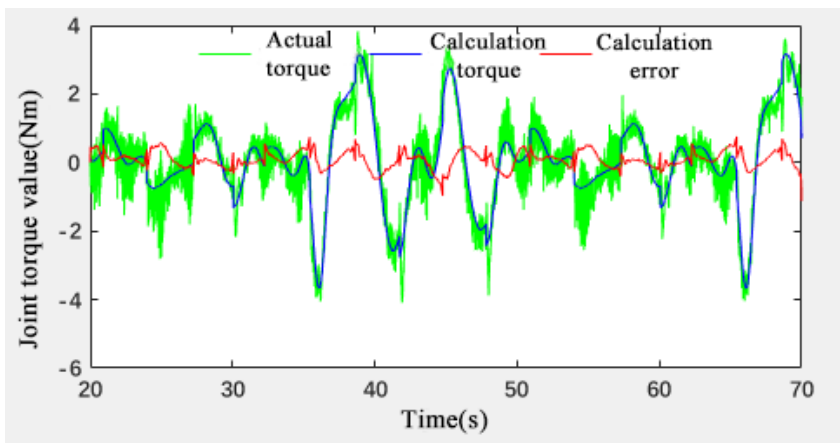

(a) Joint 1

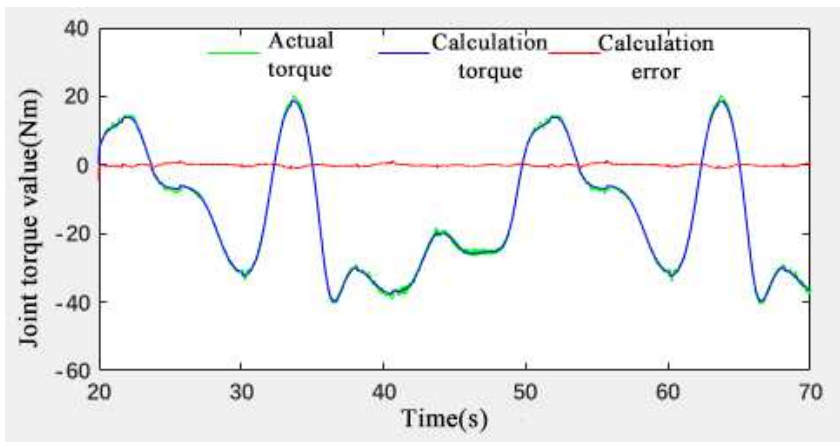

(b) Joint 2

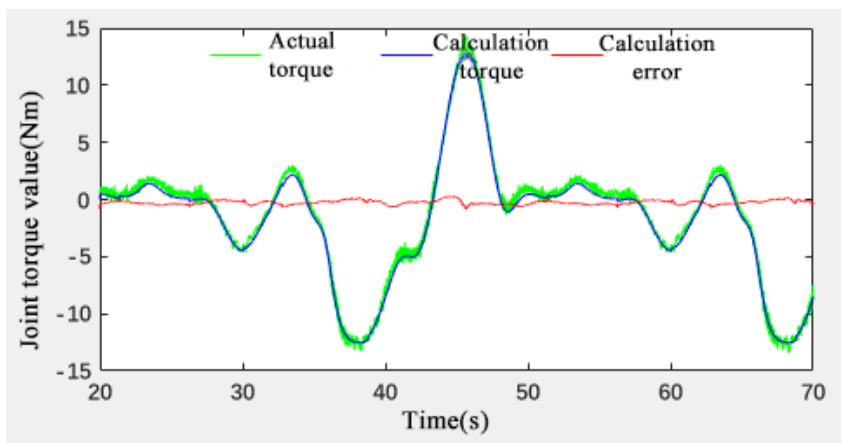

(c) Joint 3

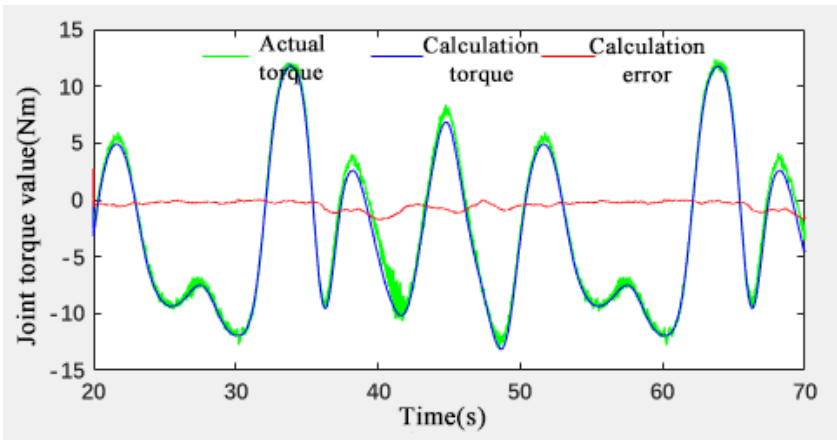

(d) Joint 4 


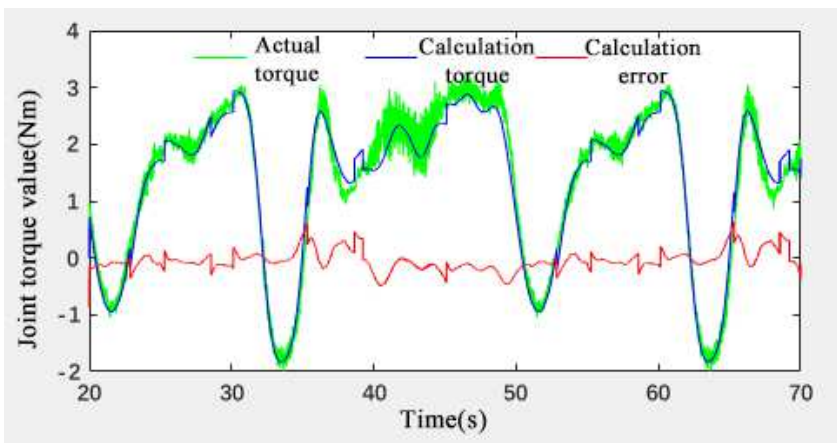

(e) Joint 5

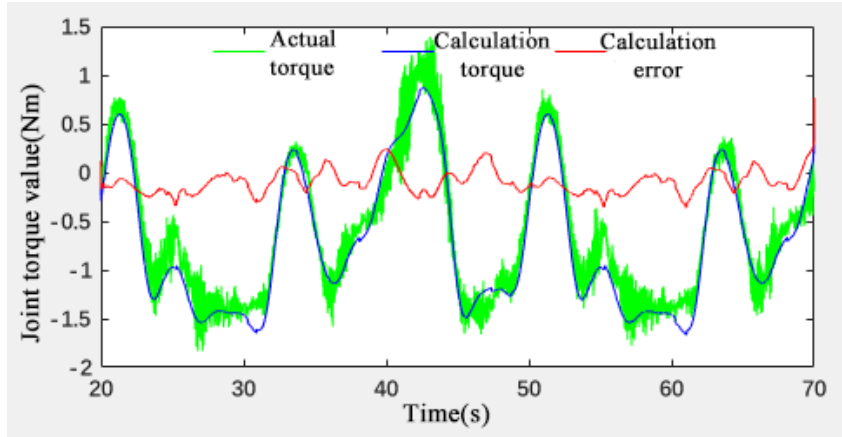

(f) Joint 6

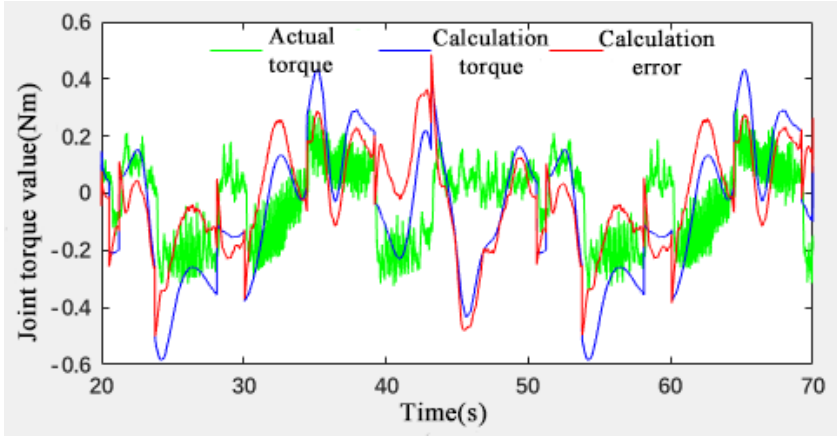

(g) Joint 7

Figure 4 inverse dynamic verification of torque values of each joint

It can be seen from the figure that the red line basically fluctuates around 0 , that is, the calculated torque value of each joint is basically consistent with the actual torque value after removing the noise, but in the joint motion, the error between the actual value near the reverse point of the joint torque and the calculated value is larger, which is consistent with the previous analysis. At the same time, because joint 7 is the end joint, the minimum joint load is only within $\pm 0.4 \mathrm{~N} / \mathrm{m}$. The error between the joint torque calculated by identification and the actual torque is large, but it has little influence in the actual work, which is consistent with the previous analysis. Generally speaking, for the dynamic parameter identification of the robotic arm, due to the large fluctuation of the acceleration signal, the identification of inertia parameters will be affected by noise signal, reverse friction and other factors, which will lead to inaccuracy near the reverse point of joint torque. Generally speaking, the parameter set identified by the linearization of the dynamic model has high accuracy and can meet the needs of practical control.

\section{Conclusions}

In this paper, the whole process of dynamic parameter identification of redundant robotic arm is studied, the spinor theory is introduced on the basis of Newton-Euler dynamic equation, the Coulomb + viscous friction model is adopted, and the minimum parameter set is further deduced by linearization. The parameter identification of the robotic arm is realized by the method of measuring current offline identification, and the excitation trajectory is designed in the basic form of Fourier series. At the same time, the joint limit and joint velocity are fully considered, and the nonlinear optimization function is used to minimize the condition number of the observation matrix as the coefficient of the objective optimization trajectory, thus the available joint trajectories are obtained. finally, the correctness and effectiveness of the method are verified on the actual robot Walker.

\section{Declaration}

\section{Acknowledgements}

Sincere thanks to Associate Professor Shi-Ping Liu for his guidance and support during the experiment and manuscript writing process.

\section{Funding}

Supported by the National Key R\&D Program of China (No. 2018YFB1306905).

\section{Availability of data and materials}

The datasets supporting the conclusions of this article are included within the article.

\section{Authors' contributions}

The author's contributions are as follows: Shi-Ping Liu was in charge of the whole trial. Jin-Liang Chen conducted experimental design and article writing; Zi-Yan $\mathrm{Ma}$ processed the data and revised the article; Other co-authors provided assistance and support during the research process

\section{Competing interests}

The authors declare no competing financial interests. 


\section{References}

[1] Claudio Urrea , José Pascal,Design, simulation, comparison and evaluation of parameter identification methods for an industrial robot Computers and Electrical Engineering,2016: 1-16.

[2] D. Zheng, Z. Xiao, and X. Xia. Multiple measurement models of articulated arm coordinate measuring machines. Chinese Journal of Mechanical Engineering (English Edition), vol. 28, no.5, pp. 994-998, 2015.

[3] A. Janot, P. C. Young, and M. Gautier. Identification and control of electro-mechanical systems using state-dependent parameter estimation. International Journal of Control, vol. 90, no. 4, pp. 643-660,2017.

[4] Gan Fangjian, Liu Zhengshi. On-line identification neural network model of inertial parameters of sensor-based operating arm. Mechanical Science and Technology, 2003(01):168-170. (in Chinese)

[5] Gregory J, Olivares A, Staffetti E. Energy - optimal trajectory planning for the Pendubot and the Acrobot. Optimal Control Applications \& Methods, 2013, 34(3):275-295.

[6] J. Jin and N. Gans. Parameter identification for industrial robots with a fast and robust trajectory design approach. Robotics and Computer-Integrated Manufacturing, vol. 31, pp. 21-29, 2015.

[7] Brunot M, Janot A, Young PC, et al. An improved instrumental variable method for industrial robot model identification. Control Eng Pract 2018; 74: 107-117.

[8] Miranda-Colorado $\mathrm{R}$ and Moreno-Valenzuela J. Experimental parameter identification of flexible joint robot manipulators. Robotica 2017; 36: 313-332.

[9] Jubien A, Gautier M, Janot A. Dynamic identification of the Kuka LightWeight robot: Comparison between actual and confidential Kuka's parameters. 2014 IEEE/ASME International Conference on Advanced Intelligent Mechatronics. IEEE, 2014: 483-488.

[10]Gaz C, De Luca A. Payload estimation based on identified coefficients of robot dynamics-With an application to collision detection. 2017 IEEE/RSJ International Conference on Intelligent Robots and Systems (IROS). IEEE, 2017: 3033-3040.

[11]Gaz C, Flacco F, De Luca A. Identifying the dynamic model used by the KUKA LWR: A reverse engineering approach. IEEE International Conference on Robotics \& Automation. 2014.

[12]E. Burdet, A. Codourey. Evaluation of parametric and nonparametric nonlinear adaptive controllers. Cambridge University Press, 1998.

[13]Honegger M , Codourey A , Burdet E . Proceedings of International Conference on Robotics and Automation - Adaptive control of the Hexaglide, a 6 dof parallel manipulator. IEEE International Conference on Robotics and Automation - Albuquerque,1997, 1:543-548.

[14]Jiang Z H , Ishida T , Sunawada M . Neural Network Aided Dynamic Parameter Identification of Robot Manipulators. IEEE International Conference on Systems, Man and Cybernetics,2006.

[15]Ghannadi B, Razavian R S, McPhee J. A modified homotopy optimization for parameter identification in dynamic systems with backlash discontinuity. Nonlinear Dynamics, 2019, 95(1): 57-72.

[16]Urrea C, Pascal J. Design, simulation, comparison and evaluation of parameter identification methods for an industrial robot. Computers \& Electrical Engineering, 2018, 67: 791-806.

[17]Tourassis V D, Neuman C P. The inertial characteristics of dynamic robot models. Mechanism and Machine Theory, 1985, 20(1):41-52.

[18]Brown P, McPhee J. A continuous velocity-based friction model for dynamics and control with physically meaningful parameters.
Journal of Computational and Nonlinear Dynamics, 2016, 11(5): 054502 .

[19]Guegan S , Khalil W , Lemoine P . Identification of the dynamic parameters of the Orthoglide. IEEE International Conference on Robotics \& Automation. IEEE, 2009.

[20]Vivas A , Poignet P , Marquet F , et al Experimental Dynamic Identification of a Fully Parallel Robot. IEEE International Conference on Robotics \& Automation. IEEE, 2003.

[21]Gautier M , Poignet P . Extended Kalman filtering and weighted least squares dynamic identification of robot. Control Engineering Practice, 2001, 9(12):1361-1372.

[22]Liu G , Iagnemma K , Dubowsky S , et al A base force/torque sensor approach to robot manipulator inertial parameter estimation. IEEE International Conference on Robotics \& Automation. IEEE, 1998.

[23]Urrea C and Pascal J. Design, simulation, comparison and evaluation of parameter identification methods for an industrial robot. Comput Electr Eng, 2016; 67: 791-806.

[24]Bahloul, A.; Tliba, S.; Chitour, Y. Dynamic Parameters Identification of an Industrial Robot with and Without Payload. IFAC-PapersOnLine, 2018, 51, 443-448.

[25]Brunot, M.; Janot, A.; Young, P.C.; Carrillo, F. An improved instrumental variable method for industrial robot model identification. Control Eng Pract. 2018, 74, 107-117.

[26]Hong B , Pey L P , Soh T Y . Structural Parameter Identification Using Robust Genetic Algorithm Optimization Method. Aiaalasme/ascelahs/asc Structures, Structural Dynamics \& Materials Conference,2013.

\section{Biographical notes}

Shi-Ping Liu, is currently an associate professor in the School of Mechanical Science and Engineering, Huazhong University of Science and Technology, China. His research interests include machine vision, intelligent robotics and Construction robotics.

Tel: +86- 027-87541034; E-mail: liushiping@ @ust.edu.cn

Zi-Yan Ma, is currently a master student in the School of Mechanical Science and Engineering, Huazhong University of Science and Technology, China.

E-mail:imaleilife@126.com

Jin-Liang Chen, is currently an engineer and he received a master's degree in mechanical engineering from Huazhong University of Science and Technology in China in 2019.

Jun-Feng Cao, is currently a master student in the School of Mechanical Science and Engineering, Huazhong University of Science and Technology, China.

Yan Fu, is currently an associate professor in the School of Mechanical Science and Engineering, Huazhong University of Science and Technology, China.

Shi-Qi Li, is currently an professor in the School of Mechanical Science and Engineering, Huazhong University of Science and Technology, China. 
Figures

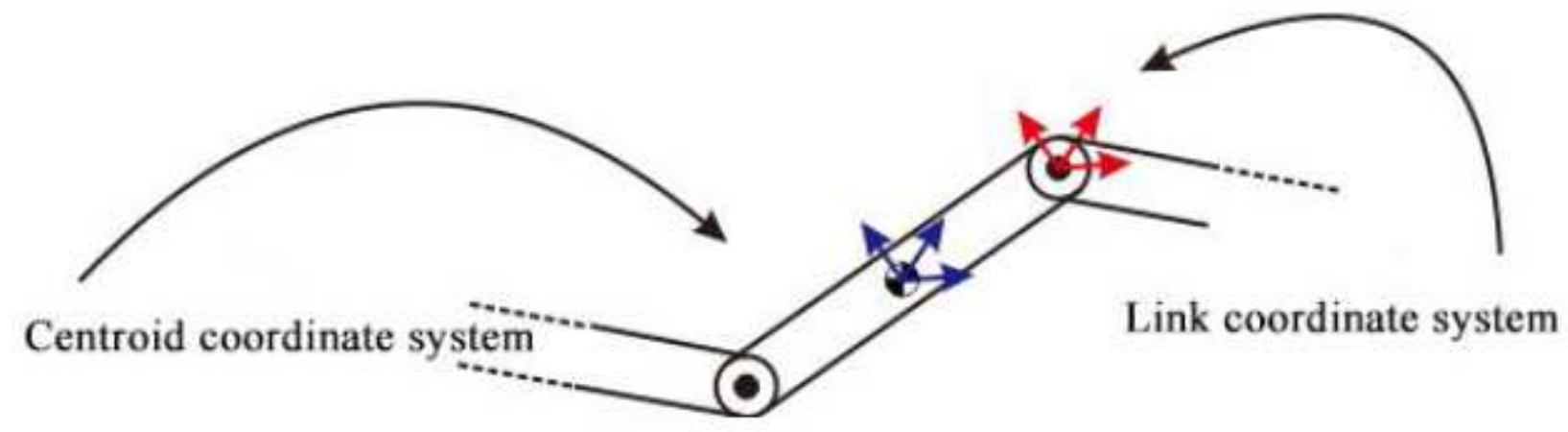

Figure 1

simplified diagram of joint parameters

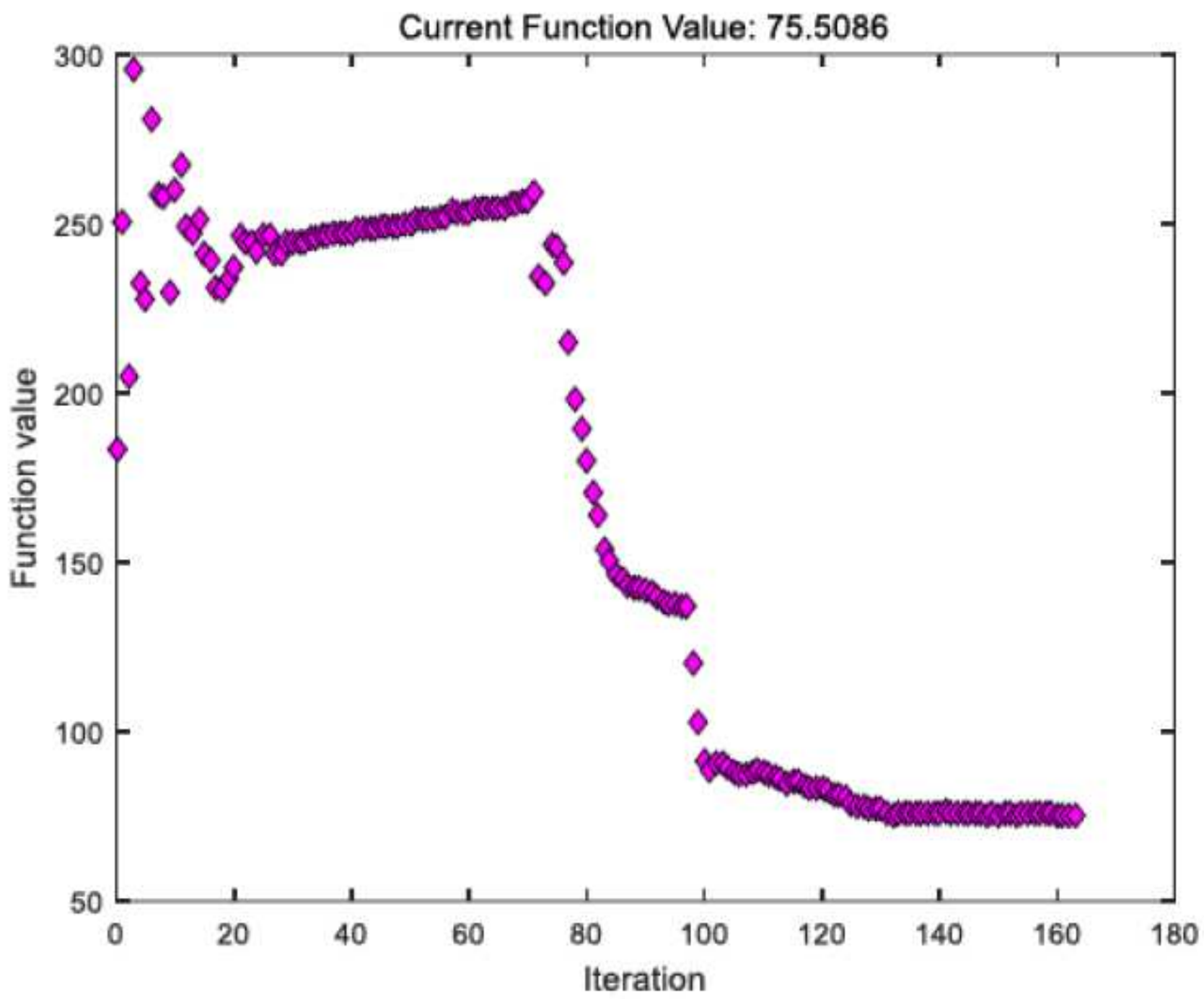

Figure 2

the change of condition number in the process of optimizing Fourier series coefficients 


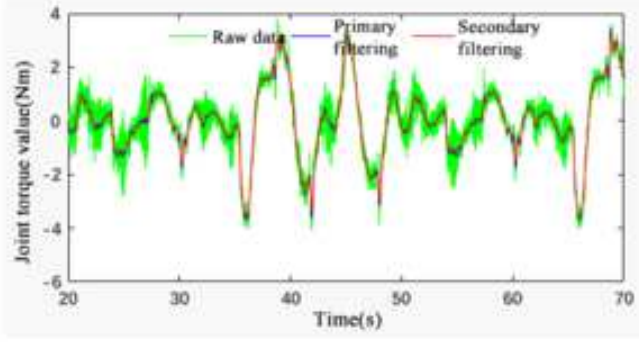

(a) Joint 1

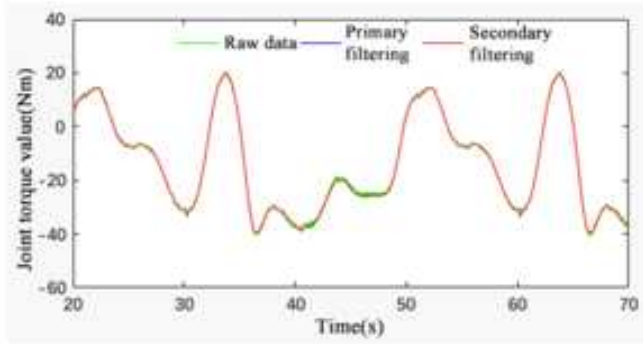

(b) Joint2

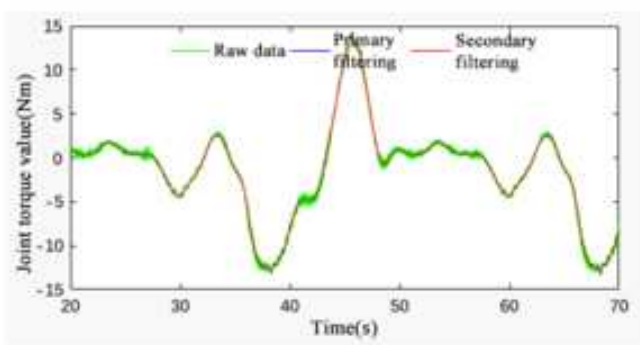

(c) Joint 3

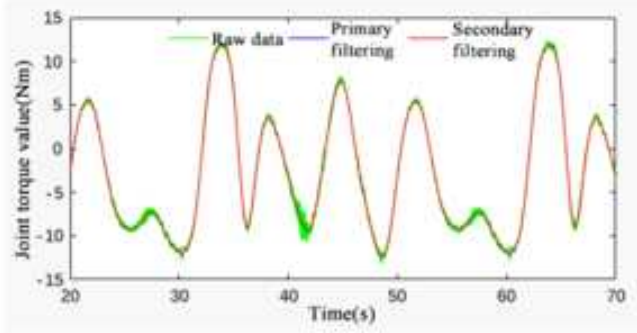

(d) Joint 4

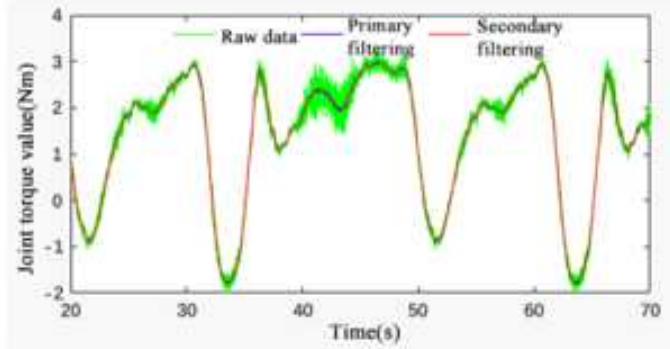

(e) Joints

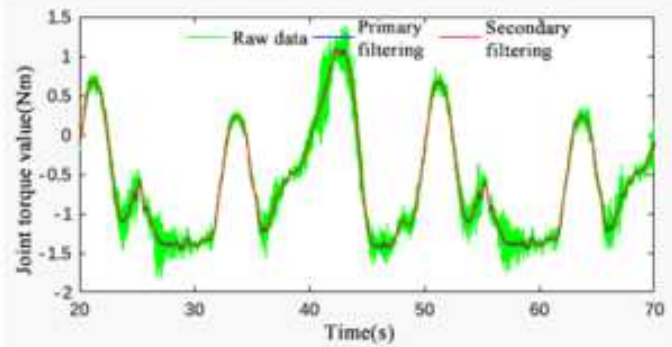

(f) Joint6

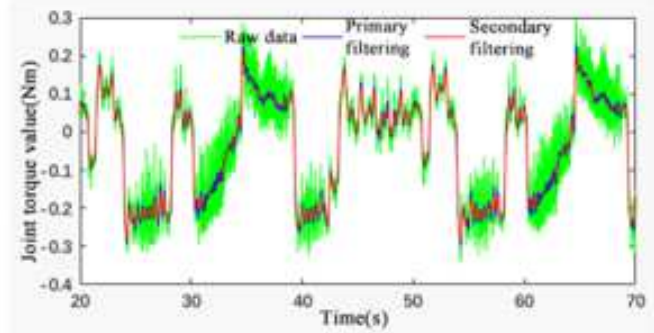

(g) Joint 7

Figure 3

joint torque value after data processing 


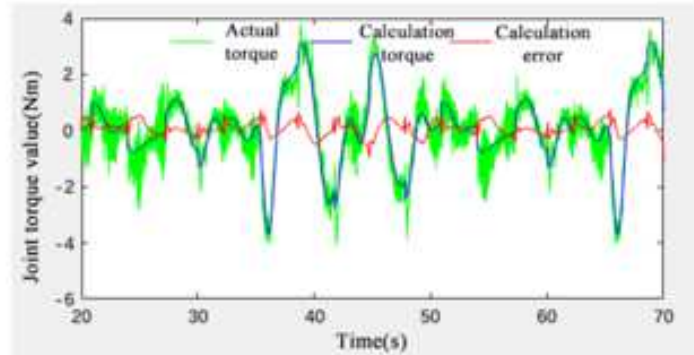

(a) Joint 1

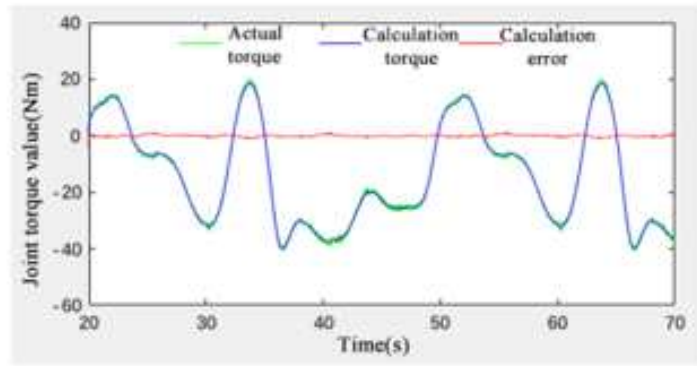

(b) Joint 2

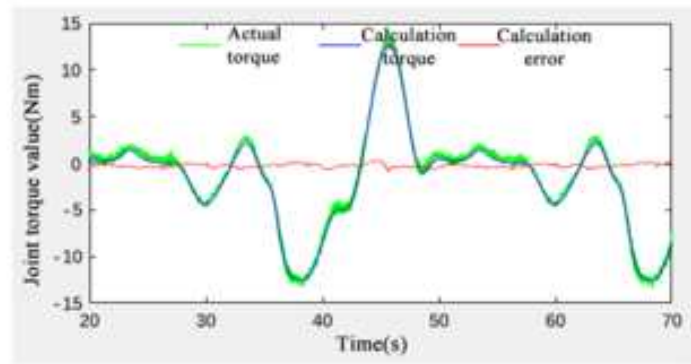

(c) Joint 3

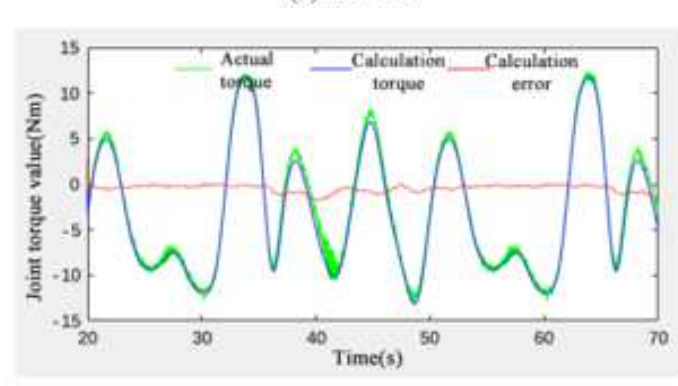

(d) Joint 4

\section{Figure 4}

inverse dynamic verification of torque values of each joint

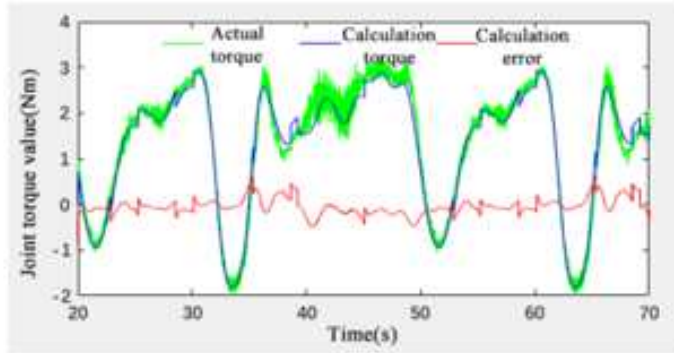

(e) Joint 5

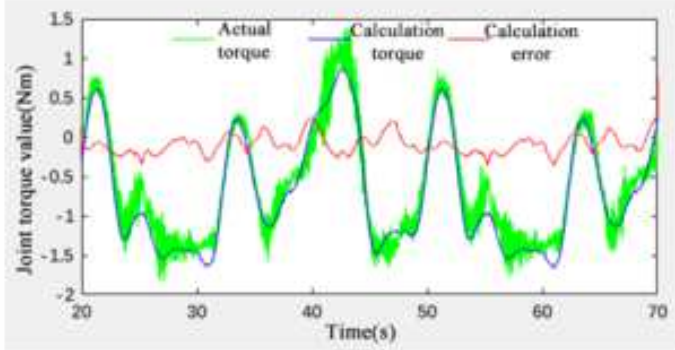

(f) Joint 6

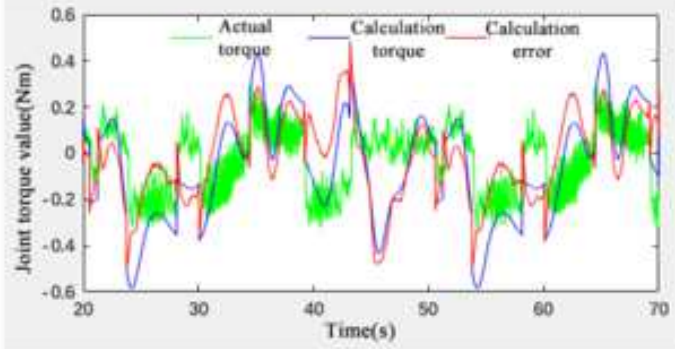

(g) Joint 7 